Check for updates

Cite this: RSC Adv., 2017, 7, 27612

Received 22nd March 2017 Accepted 12th May 2017

DOI: 10.1039/c7ra03367b

rsc.li/rsc-advances

\section{First-principles study on intrinsic defects of SnSe}

\author{
Yucheng Huang, (D) *a Chan Wang, ${ }^{a}$ Xi Chen, ${ }^{a}$ Danmei Zhou, ${ }^{a}$ Jinyan Du, ${ }^{a}$ Sufan Wang ${ }^{a}$ \\ and Lixing Ning (D) *b
}

The formation energies and electronic properties of intrinsic defects of $\mathrm{SnSe}$, including two vacancies $\left(\mathrm{V}_{\mathrm{Sn}}\right.$ and $\left.\mathrm{V}_{\mathrm{Se}}\right)$, two interstitials $\left(\mathrm{Sn}_{\mathrm{i}}\right.$ and $\left.\mathrm{Se}_{\mathrm{i}}\right)$ and two antisites $\left(\mathrm{Sn}_{\mathrm{Se}}\right.$ and $\left.\mathrm{Se}_{\mathrm{Sn}}\right)$, are investigated by using density functional theory (DFT) calculations. The results indicate that, due to a relatively low formation energy as well as a desirable ultra-shallow transition energy level, $V_{S n}$ can act as an effective source for $p$-type conduction under both $\mathrm{Sn}$ - and Se-rich conditions, which implies that SnSe is a native p-type semiconductor. On the other hand, a native $n$-type conduction is unlikely to be realized due to the absence of effective intrinsic sources. In addition, all the three types of intrinsic defects are not capable of inducing magnetism.

\section{Introduction}

As an abundant, inexpensive and environmental friendly material, tin(II) selenide has shown intriguing physical properties and wide applications in the fields of infrared optoelectronic devices, phase-change alloys for electronic memory, ${ }^{1}$ and cathodic materials for rechargeable lithium batteries. ${ }^{2}$ The structure of this important binary IV-VI group semiconductor compound crystallizes in a cubic rocksalt phase with space group $F m \overline{3} m$ or in an orthorhombic phase with space group Pmna. The two phases are comparable in thermodynamic stability and the former has been identified as a new topological crystalline insulator material., ${ }^{3,4}$ The structure of latter is similar to phosphorene ${ }^{5,6}$ and is recognized as a highly distorted $\mathrm{NaCl}$ lattice. ${ }^{7}$ Importantly, this kind of layered SnSe has a direct band gap of $1.3 \mathrm{eV}$ and an indirect band gap of $0.9 \mathrm{eV}$, falling within the range of the optimum band gap for solar cells $(1.0-1.5 \mathrm{eV}),{ }^{8}$ which makes orthorhombic SnSe an excellent candidate for application in solar cells. ${ }^{9-11}$ This has resulted in extensive studies on its synthesis and properties during recent decades. ${ }^{12-19}$ Recently, intensive interest has been devoted to the doping of foreign species in the layered SnSe, which has been shown to greatly improve electronic, magnetic and thermoelectric properties of the material. ${ }^{7,20-25}$ For example, Leng et al. ${ }^{23}$ reported that the carrier density in $\mathrm{Ag}_{x} \mathrm{Sn}_{1-x} \mathrm{Se}$ compounds was dramatically increased by substituting Sn with Ag. Wei and coworkers ${ }^{26}$

\footnotetext{
${ }^{a}$ Center for Nano Science and Technology, College of Chemistry and Material Science, The Key Laboratory of Functional Molecular Solids, Ministry of Education, Anhui Laboratory of Molecule-Based Materials, Anhui Normal University, Wuhu, 241000, Peoples' Republic of China. E-mail: huangyc@mail.ahnu.edu.cn

${ }^{b}$ Anhui Province Key Laboratory of Optoelectric Materials Science and Technology, Department of Physics, Anhui Normal University, Wuhu, 241000, Peoples' Republic of China.E-mail:ninglx@mail.ahnu.edu.cn
}

found that the carrier concentration and electrical conductivity in p-type polycrystalline SnSe were significantly enhanced in the presence of alkali metals ( $\mathrm{Li}, \mathrm{Na}$ and $\mathrm{K}$ ), among which Na displayed the best doping efficiency along with excellent thermoelectric properties. Interestingly, the first report on the thermoelectric properties of iodine-doped n-type SnSe polycrystalline demonstrated that the highest figure of merit $Z T=0.8$ can be achieved at about $773 \mathrm{~K}$ and alloying with SnS can lead to an increase of $Z T$ to 1.0. ${ }^{27} \mathrm{~A}$ very recent study reported that the presence of cubic rock-salt-type precipitate phase contributes significantly to the enhancement of electrical conductivity without the introduction of dopants. ${ }^{28}$

It was appreciated that lattice defects will be present in the material under various growth conditions, including native and exotic defects. The presence of these undesired defects could have significant effects on electronic, magnetic and optical properties of the material. Moreover, native defects could also be accompanied with chemical impurities. ${ }^{29}$ In this light, the influence of native defects should be taken into account when interpreting experimental results for material properties. To the best of our knowledge, however, no systematic investigation on the native defects in the bulk SnSe has been reported until now. Thus, there is a great need to understand the basic characteristics associated with these defects.

In the present work, we have performed DFT calculations on two vacancies $\left(\mathrm{V}_{\mathrm{Sn}}\right.$ and $\left.\mathrm{V}_{\mathrm{Se}}\right)$, two interstitials $\left(\mathrm{Sn}_{\mathrm{i}}\right.$ and $\left.\mathrm{Se}_{\mathrm{i}}\right)$, and two antisites $\left(\mathrm{Sn}_{\mathrm{Se}}\right.$ and $\left.\mathrm{Se}_{\mathrm{Sn}}\right)$ with a supercell approach. The defect formation energies, electronic and magnetic properties of these defects have been investigated. The results show that an intrinsic p-type semiconductor can be derived from $\mathrm{V}_{\mathrm{Sn}}$ in bulk SnSe under both Sn- and Se-rich conditions, whereas ntype conduction is difficult to achieve. No magnetism can be induced by all three types of intrinsic defects. 


\section{Computational methods and models}

The first-principle DFT calculations were performed with the projector augmented wave $(\mathrm{PAW})^{30}$ pseudopotentials as implemented in the Vienna $\mathrm{Ab}$ Initio Simulation Package (VASP)..$^{31-33}$ The generalized gradient approximation (GGA) with Perdew-Burke-Ernzerhof (PBE) ${ }^{34,35}$ functional was adopted to model the exchange and correlation interactions. The lattice parameters and internal ion coordinates were fully relaxed using a conjugate gradient algorithm with a force tolerance of $0.01 \mathrm{eV}^{-1}$, and electronic convergence threshold for energy was set to be $10^{-5} \mathrm{eV}$. Although a standard GGA approach would underestimate the band gap, the other properties are hardly changed as demonstrated in a recent study. ${ }^{36}$ The Monkhorst-Pack ${ }^{37} k$-point mesh of $3 \times 5 \times 5$ was employed in the first Brillouin-zone for the structural optimizations while a $3 \times 8 \times 8$ mesh was used to calculate electronic properties. A denser $6 \times 10 \times 10 k$-mesh was also used to calculate the band gap and the result only deviates by a fraction of meV from that obtained using a $3 \times 8 \times 8$ one. The cutoff energy for the plane-wave basis was $350 \mathrm{eV}$ which has been proved to be sufficient enough to give converged result. ${ }^{8}$ To determine the ground magnetic state, all systems have been examined by spin-polarized calculations.

As seen in Fig. 1a, SnSe has a double-layered geometrical structure, and the Sn and Se atoms are connected with a zigzag pattern at the same plane. The optimized lattice parameters of bulk SnSe are $a=11.64, b=4.22$ and $c=4.49 \AA$, which are in agreement with the experimental values $(a=11.55, b=4.16$, and $c=4.45 \AA$ ). ${ }^{1}$ The corresponding bond lengths of $\mathrm{Sn}$-Se were calculated to be 2.78 and $2.83 \AA$ (Fig. 1a), which also agree well with those reported in ref. 8 . In order to minimize the spurious defect-defect interactions, a $1 \times 3 \times 3$ unit cell consisting of 36 $\mathrm{Sn}$ and $36 \mathrm{Se}$ atoms was constructed from the bulk. Native defects were introduced by excavating one $\mathrm{Sn} / \mathrm{Se}$ atom $\left(\mathrm{V}_{\mathrm{Sn}} / \mathrm{V}_{\mathrm{Se}}\right)$, embedding one $\mathrm{Sn} / \mathrm{Se}$ atom $\left(\mathrm{Sn}_{\mathrm{i}} / \mathrm{Se}_{\mathrm{i}}\right)$ and substituting one $\mathrm{Sn} / \mathrm{Se}$ atom with an antisite atom $\left(\mathrm{Sn}_{\mathrm{Se}} / \mathrm{Se}\right.$ Sn $)$ (Fig. 1b). All the studied defects are proved to be the most stable one. The defect-defect distances in $y z$ plane and $x$ directions are at least $11.6 \AA$, giving a reasonable approximation of the isolated defects. To evaluate

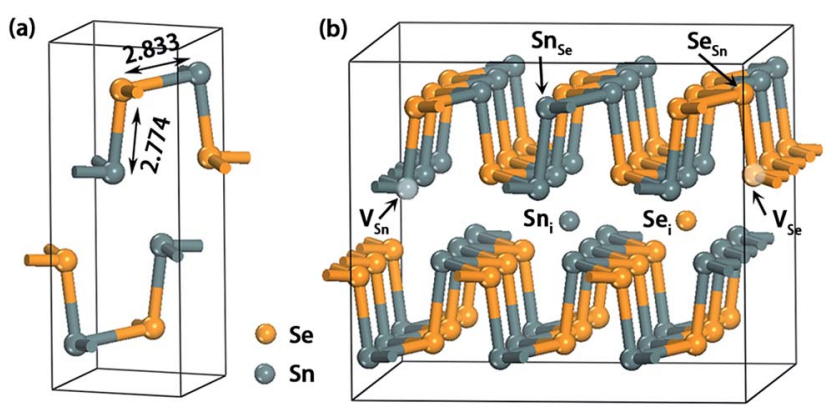

Fig. 1 (a) The geometric structure of bulk SnSe with the selected bond lengths (in Å) indicated. (b) The $1 \times 3 \times 3$ SnSe supercell structure with six intrinsic point defects under investigation is illustrated. the effect of supercell size, a larger $2 \times 4 \times 4$ supercell was constructed. First, the formation energies of vacancy defect $V_{S n}$ are calculated to be 1.53 (Sn-rich) and $0.80 \mathrm{eV}$ (Se-rich), respectively, which are slightly larger than those using a $1 \times 3 \times 3$ supercell ( 1.47 and $0.73 \mathrm{eV}$, see text). Second, the calculated transition level $\varepsilon(0 / 2-)$ of $\mathrm{V}_{\mathrm{Sn}}$ is nearly $0 \mathrm{eV}$, which is also close to the result obtained from a $1 \times 3 \times 3$ supercell ( $0.004 \mathrm{eV}$, see text). Obviously, these differences are within the error range of DFT. Note that, besides the neutral states, all point defects considered in this work are also charged in the range from $q=-2$ to +2 .

\section{Results and discussion}

\subsection{Electronic properties of the pristine $\mathrm{SnSe}$}

Before the analysis of native defects, we first investigate the electronic structure of pristine SnSe. As shown in Fig. 2a, SnSe has a band gap of $0.65 \mathrm{eV}$ at the PBE level, which is smaller than the experimental value $(0.9 \mathrm{eV}) .{ }^{38}$ Nevertheless, a band gap of $1.1 \mathrm{eV}$ was obtained when the Heyd-Scuseria-Ernzerhof (HSE06) ${ }^{39}$ screened hybrid functional was used (Fig. 2b). These results are in accordance with the previously reported values at the same computational level. ${ }^{\mathbf{8} 40}$ It can be seen from Fig. $2 \mathrm{c}$ that the lower energy valence bands in the range from -4.0 to $-2.0 \mathrm{eV}$ are ascribed to the interaction between $\mathrm{Sn}-5 \mathrm{p}$ and Se-4p states. At variance, $\mathrm{Sn}-5 \mathrm{~s}$ and $\mathrm{Sn}-5 \mathrm{p}$ states interact with Se-4p states in the upper valence bands, resulting in the formation of anti-bonding states. ${ }^{41}$ Moreover, the conduction band is mainly contributed by $\mathrm{Sn}-5 \mathrm{p}$ and Se-4p states, and Sn-5s states have almost no contribution to the conduction band minimum (CBM).

\subsection{Determination of the upper and lower limit of chemical potential}

For the calculations of defect formation energies and thermodynamic transition levels, we have simulated two different preparation conditions for these intrinsic defects, including Snrich (Se-poor) and Se-rich (Sn-poor). First, the chemical

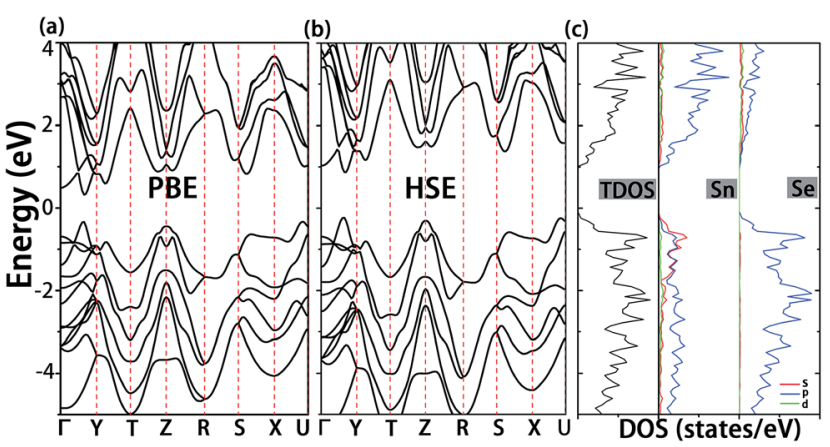

Fig. 2 Calculated band structures of the pristine SnSe at the DFT-PBE and HSE06 level are illustrated in (a) and (b), respectively. The corresponding densities of states (DOS) of the pristine SnSe by HSE06 calculation are shown in (c) and the panels from left to right are the total density of states (TDOS) and the partial density of states (PDOS) for the Sn and Se atom, respectively. 
potential of Sn or Se must be less than its natural phase to avoid the formation of hep Sn or Se crystal. As shown in Fig. 3, the upper limits for Sn and Se are indicated by vertical and parallel lines, respectively. The growth of the SnSe crystal is expected to be homogeneous if the chemical potentials of Sn and Se atoms vary within the following constraint condition,

$$
\mu_{\mathrm{SnSe}}=\mu_{\mathrm{Sn}}+\mu_{\mathrm{Se}}
$$

where $\mu_{\text {SnSe }}$ is the chemical potential of the crystal SnSe. Eqn (1) indicates the equilibrium growth condition: if the sum of $\mu_{\mathrm{Sn}}$ and $\mu_{\mathrm{Se}}$ is greater than $\mu_{\text {Snse }}$, the balance would be shifted toward left, resulting in the growth of SnSe crystal inhomogeneous; if the sum of the right terms is less than $\mu_{\text {Snse }}$, then the crystal would be decomposed. The calculated formation energy of SnSe per chemical formula is $-9.41 \mathrm{eV}$, which is represented by the blue line (Fig. 3).

Moreover, when considering alternative phases, we must set more strict limitations on the chemical potentials under thermal equilibrium growth conditions. For SnSe, another phase of $\mathrm{SnSe}_{2}$ may coexist in the system. Compared with SnSe, the atomic ration of Se to $\mathrm{Sn}$ is higher, which indicates that the $\mathrm{SnSe}_{2}$ phase tends to grow effectively under the Se-rich growth conditions. In order to suppress the formation of such an unwanted phase, the growth conditions must be restricted below the upper limit value of $\mu_{\mathrm{Se}}$. In thermodynamic equilibrium for $\mathrm{SnSe}_{2}$, the chemical potentials of $\mathrm{Sn}$ and Se atoms must satisfy the following constraint condition

$$
\mu_{\mathrm{SnSe}_{2}}=\mu_{\mathrm{Sn}}+2 \mu_{\mathrm{Se}}
$$

in which the formation energy of $\mathrm{SnSe}_{2}$ were calculated to be $\mu_{\mathrm{SnSe}_{2}}=-14.00 \mathrm{eV}$. The black line in Fig. 3 indicates the growth condition of $\mathrm{SnSe}_{2}$. It is obvious that the lines corresponding to eqn (1) and (2) cross at $\mu_{\mathrm{Sn}}=-4.82 \mathrm{eV}\left(\mu_{\mathrm{Se}}=-4.59 \mathrm{eV}\right)$. With the increase of $\mu_{\text {Se }}$, the formation energy of SnSe is higher than that of $\mathrm{SnSe}_{2}$, which means that $\mathrm{SnSe}_{2}$ will be more prone to form than SnSe under the Se-rich conditions. Consequently, $\mu_{\mathrm{Se}}=-4.59 \mathrm{eV}$ is considered as the real upper limit for the SnSe

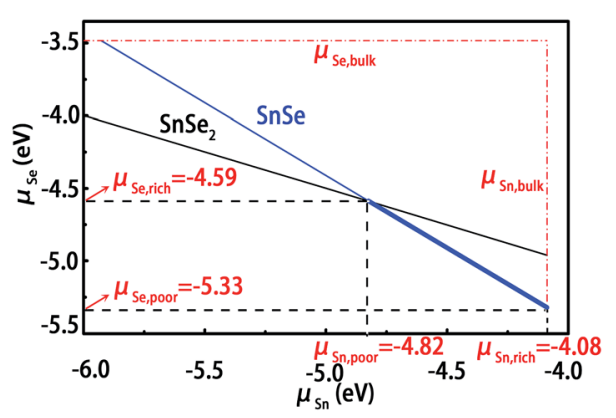

Fig. 3 Thermodynamic growth condition of SnSe crystal. The horizontal and vertical dot-dashed lines represent the upper limit of Se and $\mathrm{Sn}$ chemical potentials determined by the natural phases of Sn and Se, respectively. The blue and black solid lines depict the equilibrium growth conditions of $\mathrm{SnSe}$ and $\mathrm{SnSe}_{2}$, respectively. The range of allowable growth conditions of SnSe crystal is labelled by the bold solid line. growth. The upper and lower limits of the chemical potentials are thereby clearly labeled in Fig. 3.

\subsection{Defect formation energy}

Generally, the stabilities of different defect structures are always estimated by the calculation of the formation energy. The formation energy $\left(\Delta H_{\mathrm{D}}, q\right)$ of a defect (D) in a charge state $q$ is defined as: $:^{42}$

$$
\Delta H_{\mathrm{D}, q}=E_{\mathrm{D}, q}-E_{\mathrm{H}}-\sum_{\alpha} n_{\alpha} \mu_{\alpha}+q\left(E_{\mathrm{F}}+E_{\mathrm{V}}+\Delta V\right)
$$

where $E_{\mathrm{D}, q}$ represents the total energy of the supercell with a defect in the charge state $q$ and $E_{\mathrm{H}}$ is the total energies of the perfect supercell of the same size. $n_{\mathrm{i}}(\mathrm{i}=\mathrm{Sn}$ and Se) is the number of atoms removed from $\left(n_{\alpha}<0\right)$ or added to $\left(n_{\alpha}>0\right)$ the supercell to form a defect, $\mu_{\alpha}$ is the atomic chemical potential $^{43-45}$ and $q$ represents the number of electrons transferred from (to) electron reservoirs. ${ }^{46} E_{\mathrm{F}}$ is the Fermi energy level conventionally referenced to the energy position $\left(E_{\mathrm{V}}\right)$ of the VBM in perfect SnSe supercell. Because the VBM is referenced to the pure supercell, a correction term $\Delta V$ is used to align the electrostatic potentials between the defective and the pure supercells with the same size. This term is evaluated to be the difference between the electrostatic potential of the atomic site far from the defect in the defective supercell and that of the same site in the pure supercell. ${ }^{42}$

The formation energies of the intrinsic point defects in different charge states are listed in Table 1 . We have removed the values with formation energies larger than $1.5 \mathrm{eV}$ from the table, since these values mean low formation probabilities of the corresponding defects. As seen in Table 1, under Sn-rich

Table 1 The formation energy of defects in different charge states under the Sn-rich condition $\left(E_{\mathrm{F}, S_{n}}\right)$ and Se-rich condition $\left(E_{\left.\mathrm{F}, \mathrm{Se}_{\mathrm{e}}\right)}\right.$

\begin{tabular}{llll}
\hline Defects & $q$ & $E_{\mathrm{F}}($ Sn-rich $)$ & $E_{\mathrm{F}}($ Se-rich $)$ \\
\hline $\mathrm{V}_{\mathrm{Sn}}$ & $2-$ & 1.47 & 0.73 \\
& $1-$ & 1.42 & 0.69 \\
$\mathrm{~V}_{\mathrm{Se}}$ & 0 & 1.47 & 0.73 \\
& $2-$ & 1.95 & 2.68 \\
& $1-$ & 1.02 & 1.75 \\
& 0 & 0.16 & 0.89 \\
$\mathrm{Sn}_{\mathrm{i}}$ & $1+$ & -0.23 & 0.51 \\
& 0 & 2.48 & 3.22 \\
& $1+$ & 1.73 & 2.47 \\
$\mathrm{Se}_{\mathrm{i}}$ & $2+$ & 1.17 & 1.91 \\
& $2-$ & 5.22 & 4.49 \\
& $1-$ & 4.28 & 3.55 \\
& 0 & 3.41 & 2.67 \\
$\mathrm{Sn}_{\mathrm{Se}}$ & $1+$ & 3.24 & 2.50 \\
& $2-$ & 1.75 & 3.22 \\
& $1-$ & 1.11 & 2.58 \\
& 0 & 0.62 & 2.09 \\
& $1+$ & 0.39 & 1.86 \\
$\mathrm{Se}_{\mathrm{Sn}}$ & $2+$ & 0.17 & 1.65 \\
& $2-$ & 4.11 & 2.64 \\
& 0 & 3.41 & 1.94 \\
& $1+$ & 3.21 & 1.74 \\
& $2+$ & 3.15 & 1.68
\end{tabular}


condition, the three defects in their neutral charge states $\left(\mathrm{Sn}_{\mathrm{Se}}\right.$, $\mathrm{V}_{\mathrm{Sn}}$ and $\mathrm{V}_{\mathrm{Se}}$ ) have the potential to affect SnSe electrical properties due to their relatively low formation energies $(<1.5 \mathrm{eV})$. Under Se-rich condition, the formation energies of the neutral vacancies are still relatively low. It is interesting to observe that different preparation conditions have different effects on the formation energies: $\mathrm{V}_{\mathrm{Se}}, \mathrm{Sn}_{\mathrm{i}}$ and $\mathrm{Sn}_{\mathrm{Se}}$ have lower formation energies under Sn-rich condition, but under Se-rich condition, the formation energies of $\mathrm{V}_{\mathrm{Sn}}, \mathrm{Se}_{\mathrm{i}}$ and $\mathrm{Se}_{\mathrm{Sn}}$ become lower instead. Therefore, compared with cation-poor condition, cation interstitials and anion vacancies are more energetically favorable under the anion-poor condition. On the contrary, anion interstitials and cation vacancies are prone to be formed under the cation-poor condition. Similar findings have been reported in the studies of native defects in $\mathrm{ZnO}^{47}$ and $\mathrm{SnS}^{48}$

Defects often introduce levels in the band gap or near the band edge. ${ }^{49}$ The transition level of a defect is the Fermi level position where the defect can donate/accept electrons. Deep level means that removing an electron or hole from the trap to the valance or conduction band requires relative high energy. The transition level $\varepsilon\left(q / q^{\prime}\right)$ is defined as the Fermi level position for which the formation energies of a defect in charge states $q$ is equal to that in charge state $q^{\prime}$, which can be expressed as ${ }^{42}$

$$
\varepsilon\left(q / q^{\prime}\right)=\frac{E_{\mathrm{F}}\left(\operatorname{SnSe}^{q}\right)-E_{\mathrm{F}}\left(\operatorname{SnSe}^{q^{\prime}}\right)}{q-q^{\prime}} .
$$

Fig. 4a and $\mathrm{b}$ present the calculated formation energies of the six fundamental intrinsic defects in SnSe as a function of the Fermi level $\left(E_{\mathrm{F}}\right)$ under the Sn- and Se-rich conditions, respectively. Here the charged states from $q=-2$ to +2 are considered for the charged defects since the nominal valence of $\mathrm{Sn} / \mathrm{Se}$ in a perfect $\mathrm{SnSe}$ is $\mathrm{Sn}^{2+} / \mathrm{Se}^{2-}$. For vacancies, under both conditions, it can be easily identified that $\mathrm{V}_{\mathrm{Sn}}$ with $q=-2$ has the lowest formation energy among all kinds of intrinsic defects and a desirable ultra-shallow transition energy level $\varepsilon(0 / 2-)$ located very closely to the VBM. This indicates that $\mathrm{V}_{\mathrm{Sn}}$ can act as a shallow acceptor which is likely to make a prominent contribution to p-type conduction. With the increase of hole carriers concentration, $E_{\mathrm{F}}$ will move downward and then stay on
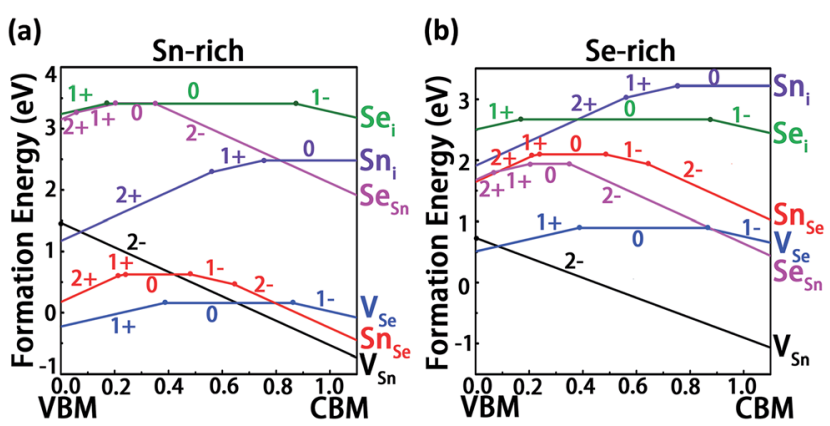

Fig. 4 The calculated formation energies of intrinsic defects in SnSe as a function of Fermi level $\left(E_{\mathrm{F}}\right)$ under the Sn-rich (a) and Se-rich conditions (b). The band gap value is the one calculated by DFT-HSE06 method. the crossing point between $\mathrm{V}_{\mathrm{Sn}}(q=-2)$ and $\mathrm{V}_{\mathrm{Se}}(q=0)\left(\mathrm{V}_{\mathrm{Se}}(q=\right.$ $+1)$ ) under Sn-rich (Se-rich) condition (Fig. 4). In view of the fact that $\mathrm{V}_{\mathrm{Sn}}$ is more energetically favorable than $\mathrm{V}_{\mathrm{Se}}$ under the Serich condition, this condition is thereby favorable for the formation of $\mathrm{V}_{\mathrm{Sn}}$. Nevertheless, it is also important to be aware of the formation of other Se-rich phase like $\mathrm{SnSe}_{2}$ during the growth. Thus, manipulating the growth condition not toward Se-rich too much is required for preventing the formation of $\mathrm{SnSe}_{2}$ phase. Similar to $\mathrm{V}_{\mathrm{Sn}}, \mathrm{V}_{\mathrm{Se}}$ also has the relative low formation energy under both growth conditions. However, the transition energy level $\varepsilon(1+/ 0)$ is closer to the VBM rather than the CBM, such that the $\mathrm{V}_{\mathrm{Se}}$ defect is in a stable neutral charge state at high $E_{\mathrm{F}}(\geq 0.384 \mathrm{eV})$ and acts as a deep donor. Accordingly, $\mathrm{V}_{\mathrm{Se}}$ is predicted to have an insignificant effect on $\mathrm{n}$-type conduction. For the case of antisite defects, the transition energy level $\varepsilon(1+/ 0)$ of $\mathrm{Sn}_{\mathrm{Se}}$ lies far away from the CBM, which means that it will become positively ionized only when Fermi level is in the low energy region $E_{\mathrm{F}} \leq 0.237$, and thus $\mathrm{Sn}_{\mathrm{Se}}$ acts as a deep donor and is excluded from appropriate candidates for $\mathrm{p}$ type conduction. $\mathrm{Se}_{\mathrm{Sn}}$ is a deep acceptor with a high positive formation energy, which indicates $\mathrm{Se}_{\mathrm{Sn}}$ is not an effective source for n-type conduction. In terms of interstitial defects, the donor defect $\mathrm{Sn}_{\mathrm{i}}$ has a high transition energy level $\varepsilon(1+/ 0)$ with respect to the CBM, however the high positive formation energies of $\mathrm{Sn}_{\mathrm{i}}$ under both conditions indicate that this shallow donor defect is unlikely to form, with minor contribution to n-type conduction. $\mathrm{Se}_{\mathrm{i}}$ can act as an acceptor but it has an ultra-deep transition energy level which is localized extremely near the edge of CBM. Moreover, this defect is energetically unfavorable and thus its contribution to the p-type conduction may be ignored. Although the contribution to n-type conduction could be expected, due to the relative low $\varepsilon(1+/ 0)$ transition level with respect to the VBM, the thermodynamic instability determines that it is not the main source (Table 2).

From the above analysis, we can conclude that no intrinsic defect can act as an effective source for n-type conduction under both growth conditions. On the other hand, p-type conduction can be easily achieved by introducing $\mathrm{V}_{\mathrm{Sn}}$ in SnSe, which

Table 2 The transition energy levels $\varepsilon\left(q / q^{\prime}\right)$ referenced to VBM for intrinsic defects in SnSe

\begin{tabular}{lll}
\hline Defects & $q / q^{\prime}$ & $\varepsilon\left(q / q^{\prime}\right)(\mathrm{eV})$ \\
\hline $\mathrm{V}_{\mathrm{Sn}}$ & $2-/ 0$ & 0.004 \\
$\mathrm{~V}_{\mathrm{Se}}$ & $0 / 1-$ & 0.86 \\
& $1+/ 0$ & 0.38 \\
$\mathrm{Sn}_{\mathrm{i}}$ & $1+/ 0$ & 0.75 \\
& $2+/ 1+$ & 0.56 \\
$\mathrm{Se}_{\mathrm{i}}$ & $0 / 1-$ & 0.87 \\
& $1+/ 0$ & 0.17 \\
$\mathrm{Sn}_{\mathrm{Se}}$ & $1-/ 2-$ & 0.64 \\
& $0 / 1-$ & 0.48 \\
& $1+/ 0$ & 0.24 \\
& $2+/ 1+$ & 0.21 \\
$\mathrm{Se}_{\mathrm{Sn}}$ & $0 / 2-$ & 0.35 \\
& $1+/ 0$ & 0.20 \\
& $2+/ 1+$ & 0.06
\end{tabular}


suggests that SnSe is an intrinsic p-type semiconductor. This result agrees well with the reported observations that the bulk SnSe exhibits a p-type conductivity with hole mobilities up to $10^{-3} \mathrm{~cm}^{2} \mathrm{~V}^{-1} \mathrm{~s}^{-1}$ along the $c$ axis..$^{50,51}$

\subsection{Electronic properties of intrinsic defects}

To further validate the above conclusions, the effects of intrinsic defects on electronic and magnetic properties of SnSe have also been studied. Spin-polarized calculations were first carried out to examine whether the intrinsic defects can induce magnetisms into the system. Unlike versatile magnetic properties of $\mathrm{GaS}^{52}$ and $\mathrm{MoS}_{2}$ (ref. 53) monolayers induced by native defects, no magnetism was found for the intrinsic defects in SnSe, which is evidenced by the observation that the DOSs for the majority and minority spin channels are symmetrical (Fig. 5). For the Sn vacancy, the DOS near the valance band are mainly contributed by Se-4p states. Meanwhile, Sn-5p states play an important role in the states near the CBM. The Fermi level shifts toward the valance band, rendering the material a p-type characteristic. For $\mathrm{V}_{\mathrm{Se}}$, a small narrow peak appears around the VBM which is mainly contributed by $\mathrm{Sn}-5 \mathrm{p}$ states. The $\mathrm{V}_{\mathrm{Se}}$ defect is a deep donor as indicated above, thus it is not an effective source for n-type conduction. For the case of antisite defects,
$\mathrm{Sn}_{\text {Se }}$ acts as a deep donor while $\mathrm{Se}_{\mathrm{Sn}}$ acts as a deep acceptor. In the case of interstitial defects, an extra Sn atom causes the concentration of electrons to increase, resulting in an upward movement of $E_{\mathrm{F}}$. It is clearly seen that the DOS near the CBM are mainly contributed by $\mathrm{Sn}-4 \mathrm{p}$ states, with minor contribution from $S n-4 s . S_{i}$ defect could act as a shallow donor, but the high formation energy excluded its role in p-type conduction. On the other hand, as seen from Fig. 5, both the $\varepsilon(1+/ 0)$ and $\varepsilon(0 / 1-)$ transition levels of the $\mathrm{Se}_{\mathrm{i}}$ are far from the CBM and VBM, thus failing to contribute the $\mathrm{n}$ - and p-type conductivities.

It is worth noting that the long range van der Waals interactions maybe play a pivotal role in the layered structure. Therefore, the PBE-D3 method $^{54}$ including the dispersion interaction between two layers is carefully checked. The PBE-D3 optimized lattice parameters $(a=11.64, b=4.21$ and $c=4.49 \AA)$ are essentially the same to those by PBE one, and the calculated band gap of bulk SnSe is $0.66 \mathrm{eV}$, also close to the corresponding $\mathrm{PBE}$ value. To further validate the effect on other conclusion we made, we choose $\mathrm{V}_{\mathrm{Sn}}$ to recalculate its transition levels. Our results showed that the $\varepsilon(0 / 2-)$ of $\mathrm{V}_{\mathrm{Sn}}$ is $0.02 \mathrm{eV}$, demonstrating that adding " $D$ " has minor effect on the main conclusions. In fact, previous studies ${ }^{48,55}$ regarding on intrinsic defects of the same layered materials also ignored the dispersion effect.
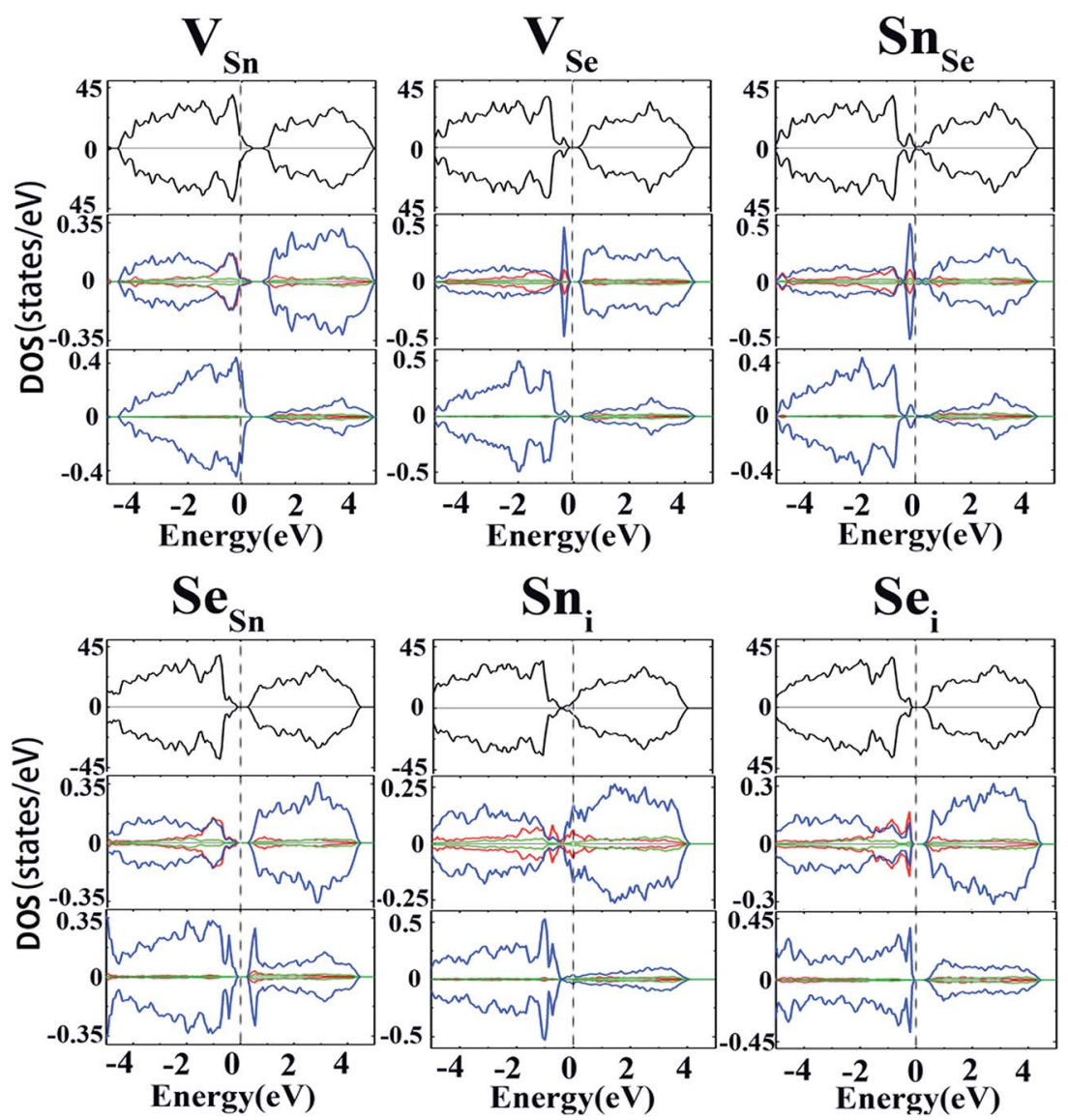

Fig. 5 Calculated TDOSs and PDOSs for the intrinsic defects $\left(V_{S n}, V_{S_{e}}, S n_{i}, S e_{i}, S n_{S e}\right.$ and $\left.S e_{S n}\right)$ in $S n S e$ (at the PBE level). The panels from top to bottom are the TDOS, PDOS of the nearest $\mathrm{Sn}$ atom and Se atom to the defect position, respectively. The $\mathrm{s}$, $\mathrm{p}$ and $\mathrm{d}$ orbitals are marked up with red, blue and green lines, respectively. The vertical lines show the position of $E_{\mathrm{F}}$. 


\section{Conclusion}

In conclusion, the formation energies, electronic and magnetic properties of six intrinsic defects in SnSe were systematically investigated on the basis of DFT. It was shown that $\mathrm{V}_{\mathrm{Sn}}$ is able to make a prominent contribution to the p-type conduction under both Sn- and Se-rich conditions due to its relatively lower formation energy and a desirable ultra-shallow thermodynamic transition level. By contrast, intrinsic n-type conduction was difficult to achieve since no effective sources were found. Our results also revealed that all intrinsic defects are unable to induce magnetism. Overall, this work provides a good start to the realization of p-type conduction in SnSe in the absence of impurities.

\section{Acknowledgements}

This work was supported by National Natural Science Foundation of China No. 21573002 (H. Y.), 11574003 (N. L) and 21373012 (W. S.). The numerical calculations in this paper have been done on the super-computing system in the Supercomputing Center of University of Science and Technology of China.

\section{References}

1 M. A. Franzman, C. W. Schlenker, M. E. Thompson and R. L. Brutchey, J. Am. Chem. Soc., 2010, 132, 4060-4061.

2 J. Ning, G. Xiao, T. Jiang, L. Wang, Q. Dai, B. Zou, B. Liu, Y. Wei, G. Chen and G. Zou, CrystEngComm, 2011, 13, 4161-4166.

3 Y. Sun, Z. Zhong, T. Shirakawa, C. Franchini, D. Li, Y. Li, S. Yunoki and X.-Q. Chen, Phys. Rev. B: Condens. Matter Mater. Phys., 2013, 88, 235122.

4 Z. Wang, J. Wang, Y. Zang, Q. Zhang, J.-A. Shi, T. Jiang, Y. Gong, C.-L. Song, S.-H. Ji, L.-L. Wang, L. Gu, K. He, W. Duan, X. Ma, X. Chen and Q.-K. Xue, Adv. Mater., 2015, 27, 4150-4154.

5 G. Shi and E. Kioupakis, J. Appl. Phys., 2015, 117, 065103.

6 T. Chattopadhyay, J. Pannetier and H. Von Schnering, J. Phys. Chem. Solids, 1986, 47, 879-885.

7 S. Chen, K. Cai and W. Zhao, Phys. B, 2012, 407, 4154-4159.

8 Y. Huang, C. Ling, H. Liu and S. Wang, RSC Adv., 2014, 4, 6933-6938.

9 W. J. Baumgardner, J. J. Choi, Y.-F. Lim and T. Hanrath, J. Am. Chem. Soc., 2010, 132, 9519-9521.

10 S. Liu, X. Guo, M. Li, W.-H. Zhang, X. Liu and C. Li, Angew. Chem., Int. Ed., 2011, 50, 12050-12053.

11 P. D. Antunez, J. J. Buckley and R. L. Brutchey, Nanoscale, 2011, 3, 2399-2411.

12 A. J. Hong, L. Li, H. X. Zhu, Z. B. Yan, J. M. Liu and Z. F. Ren, J. Mater. Chem. A, 2015, 3, 13365-13370.

13 S. Zhao, H. Wang, Y. Zhou, L. Liao, Y. Jiang, X. Yang, G. Chen, M. Lin, Y. Wang, H. Peng and Z. Liu, Nano Res., 2015, 8, 288-295.

14 K.-M. Chung, D. Wamwangi, M. Woda, M. Wuttig and W. Bensch, J. Appl. Phys., 2008, 103, 083523.
15 L.-D. Zhao, S.-H. Lo, Y. Zhang, H. Sun, G. Tan, C. Uher, C. Wolverton, V. P. Dravid and M. G. Kanatzidis, Nature, 2014, 508, 373-377.

16 L. Li, Z. Chen, Y. Hu, X. Wang, T. Zhang, W. Chen and Q. Wang, J. Am. Chem. Soc., 2013, 135, 1213-1216.

17 B. Nariya, A. Dasadia, M. Bhayani, A. Patel and A. Jani, Chalcogenide Lett., 2009, 6, 549-554.

18 C. W. Li, J. Hong, A. F. May, D. Bansal, S. Chi, T. Hong, G. Ehlers and O. Delaire, Nat. Phys., 2015, 11, 1063-1065.

19 Y. C. Huang, X. Chen, C. Wang, L. Peng, Q. Qian and S. F. Wang, Nanoscale, 2017, DOI: 10.1039/C7NR01952A.

20 Y. Huang, C. Ling, Z. Fang and S. Wang, Phys. E, 2014, 59, 102-106.

21 E. K. Chere, Q. Zhang, K. Dahal, F. Cao, J. Mao and Z. Ren, J. Mater. Chem. A, 2016, 4, 1848-1854.

22 Q. Wang, W. Yu, X. Fu, C. Qiao, C. Xia and Y. Jia, Phys. Chem. Chem. Phys., 2016, 18, 8158-8164.

23 H. Leng, M. Zhou, J. Zhao, Y. Han and L. Li, J. Electron. Mater., 2016, 45, 527-534.

24 K. Peng, X. Lu, H. Zhan, S. Hui, X. Tang, G. Wang, J. Dai, C. Uher, G. Wang and X. Zhou, Energy Environ. Sci., 2016, 9, 454-460.

25 N. K. Singh, S. Bathula, B. Gahtori, K. Tyagi, D. Haranath and A. Dhar, J. Alloys Compd., 2016, 668, 152-158.

26 T.-R. Wei, G. Tan, X. Zhang, C.-F. Wu, J.-F. Li, V. P. Dravid, G. J. Snyder and M. G. Kanatzidis, J. Am. Chem. Soc., 2016, 138, 8875-8882.

27 Q. Zhang, E. K. Chere, J. Sun, F. Cao, K. Dahal, S. Chen, G. Chen and Z. Ren, Adv. Energy Mater., 2015, 5, 1500360.

28 G. Tang, Q. Wen, T. Yang, Y. Cao, W. Wei, Z. Wang, Z. Zhang and Y. Li, RSC Adv., 2017, 7, 8258-8263.

29 S. Na-Phattalung, M. F. Smith, K. Kim, M.-H. Du, S.-H. Wei, S. B. Zhang and S. Limpijumnong, Phys. Rev. B: Condens. Matter Mater. Phys., 2006, 73, 125205.

30 P. E. Blöchl, Phys. Rev. B: Condens. Matter Mater. Phys., 1994, 50, 17953-17979.

31 G. Kresse and J. Hafner, Phys. Rev. B: Condens. Matter Mater. Phys., 1994, 49, 14251-14269.

32 G. Kresse and J. Hafner, Phys. Rev. B: Condens. Matter Mater. Phys., 1993, 47, 558-561.

33 G. Kresse and J. Furthmüller, Comput. Mater. Sci., 1996, 6, 15-50.

34 J. P. Perdew and Y. Wang, Phys. Rev. B: Condens. Matter Mater. Phys., 1992, 45, 13244-13249.

35 J. P. Perdew, J. A. Chevary, S. H. Vosko, K. A. Jackson, M. R. Pederson, D. J. Singh and C. Fiolhais, Phys. Rev. B: Condens. Matter Mater. Phys., 1992, 46, 6671-6687.

36 S. Prada, L. Giordano and G. Pacchioni, J. Phys. Chem. C, 2013, 117, 9943-9951.

37 H. J. Monkhorst and J. D. Pack, Phys. Rev. B: Solid State, 1976, 13, 5188-5192.

38 I. Lefebvre, M. A. Szymanski, J. Olivier-Fourcade and J. C. Jumas, Phys. Rev. B: Condens. Matter Mater. Phys, 1998, 58, 1896-1906.

39 J. Heyd, G. E. Scuseria and M. Ernzerhof, J. Chem. Phys., 2006, 124, 219906. 
40 L. C. Gomes and A. Carvalho, Phys. Rev. B: Condens. Matter Mater. Phys., 2015, 92, 085406.

41 J. Yang, G. Zhang, G. Yang, C. Wang and Y. X. Wang, J. Alloys Compd., 2015, 644, 615-620.

42 C. G. Van de Walle and J. Neugebauer, J. Appl. Physiol., 2004, 95, 3851-3879.

43 C. Persson, Y.-J. Zhao, S. Lany and A. Zunger, Phys. Rev. B: Condens. Matter Mater. Phys., 2005, 72, 035211.

44 D. B. Laks, C. G. Van de Walle, G. F. Neumark, P. E. Blöchl and S. T. Pantelides, Phys. Rev. B: Condens. Matter Mater. Phys., 1992, 45, 10965-10978.

45 S. B. Zhang and J. E. Northrup, Phys. Rev. Lett., 1991, 67, 2339-2342.

46 S.-H. Wei, Comput. Mater. Sci., 2004, 30, 337-348.

47 A. Janotti and C. G. Van de Walle, Phys. Rev. B: Condens. Matter Mater. Phys., 2007, 76, 165202.
48 Z. Xiao, F.-Y. Ran, H. Hosono and T. Kamiya, Appl. Phys. Lett., 2015, 106, 152103.

49 Y. Huang, D. Zhou, X. Chen, H. Liu, C. Wang and S. Wang, ChemPhysChem, 2016, 17, 375-379.

50 H. Maier and D. R. Daniel, J. Electron. Mater., 1977, 6, 693704.

51 J.-i. Umeda, J. Phys. Soc. Jpn., 1961, 16, 124-125.

52 H. Chen, Y. Li, L. Huang and J. Li, RSC Adv., 2015, 5, 5088350889.

53 D. Cao, H. B. Shu, T. Q. Wu, Z. T. Jiang, Z. W. Jiao, M. Q. Cai and W. Y. Hu, Appl. Surf. Sci., 2016, 361, 199-205.

54 S. Grimme, J. Antony, S. Ehrlich and H. Krieg, J. Chem. Phys., 2010, 132, 154104.

55 A. Togo, F. Oba, I. Tanaka and K. Tatsumi, Phys. Rev. B: Condens. Matter Mater. Phys., 2006, 74, 195128. 